\title{
Measurements of stress-optic coefficient and Young's modulus in PMMA fibers drawn under different conditions
}

\author{
Marcin K. Szczurowski ${ }^{1}$, Tadeusz Martynkien ${ }^{1}$, Gabriela Statkiewicz-Barabach ${ }^{1}$, Lutful Khan ${ }^{2}$, \\ David J. Webb ${ }^{2}$, Chenchun Ye ${ }^{3}$, Janice Dulieu-Barton ${ }^{3}$, Waclaw Urbanczyk ${ }^{1, *}$ \\ ${ }^{1}$ Institute of Physics, Wroclaw Univ. of Technology, Wyb. Wyspianskiego 27, 50-370 Wroclaw, \\ Poland, *waclaw.urbanczyk@.pwr.wroc.pl \\ ${ }^{2}$ Photonics Research Group, Aston University, Birmingham, B4 7ET, UK \\ ${ }^{3}$ Univ. of Southampton, School of Engineering Sciences, Highfield, Southampton, SO17 1BJ, UK
}

\begin{abstract}
We have systematically measured the differential stress-optic coefficient, $\Delta \mathrm{C}$, and Young's modulus, $\mathrm{E}$, in a number of PMMA fibers drawn with different stress, ranging from 2 up to $27 \mathrm{MPa}$. Effect of temperature annealing on those parameters was also investigated. $\Delta \mathrm{C}$ was determined in transverse illumination by measuring the dependence of birefringence on additional axial stress applied to the fiber. Our results show that $\Delta \mathrm{C}$ in PMMA fibers has a negative sign and ranges from -4.5 to $-1.5 \times 10^{-12} \mathrm{~Pa}^{-1}$ depending on the drawing stress. Increase of the drawing stress results in greater initial fiber birefringence and lower $\Delta \mathrm{C}$. The dependence of $\Delta \mathrm{C}$ and initial birefringence upon drawing stress is nonlinear and gradually saturates for higher drawing stress. Moreover, we find that $\Delta \mathrm{C}$ is linearly proportional to initial fiber birefringence and that annealing the fiber has no impact on the slope of this dependence. On the other hand, no clear dependence was observed between the fiber drawing stress and the Young's modulus of the fibers as measured using microscopic digital image correlation with the fibers tensioned using an Instron tension tester.
\end{abstract}

Keywords: polymer fibers, birefringence, stress-optic coefficient, Young's modulus

\section{INTRODUCTION}

PMMA is a very popular polymer widely used in photonic technology for fabrication of micro-optical elements, integrated devices and optical fibers. There are many literature inconsistencies concerning its mechanical constants, especially the differential stress-optic coefficient $\Delta \mathrm{C}=\mathrm{C}_{1}-\mathrm{C}_{2}$. Measurements performed in PMMA discs (18mm diameter, $10 \mathrm{~mm}$ thick) showed $\Delta \mathrm{C}$ equal to $-3.3 \times 10^{-12} \mathrm{~Pa}^{-1}$ [1], but in [2] the measurements carried out in discs of different size (13.03 mm diameter, $2.85 \mathrm{~mm}$ thick) gave a value of $\Delta \mathrm{C}=5.5 \times 10^{-10} \mathrm{~Pa}^{-1}$. Another quite different result was reported in [3], $\Delta \mathrm{C}=1.08 \times 10^{-10} \mathrm{~Pa}^{-1}$. Very systematic studies of the optical and mechanical properties of bulk PMMA (Plexiglas 55) are presented in [4]. In particular, the strain-optic coefficients for this material have been measured $p_{11}=0.300, p_{12}=0.297$ in [4], which leads to a calculated value of $\Delta \mathrm{C}=-2.30 \times 10^{-12} \mathrm{~Pa}^{-1}$, assuming the following material constants: refractive index $n=1.4904$ at a wavelength $\lambda=690 \mathrm{~nm}$, Young's modulus E=3 GPa, and Poisson's ratio $v=0.37$.

The purpose of this study is to measure systematically the differential stress-optic coefficient and Young's modulus in a set of PMMA fibers drawn under different conditions. The fibers used in this study were all drawn from homogeneous, nominally pure PMMA rods obtained from AMARI plastics. The mean molecular weight was determined by gas permeation chromatography to be 87,000 . To prevent the preforms from bubbling in the draw tower oven, they were annealed at $80^{\circ} \mathrm{C}$ for 48 hours immediately prior to fiber drawing. The fibers were all drawn with a hot-zone temperature of $181 \pm 2^{\circ} \mathrm{C}$ and the fiber tension controlled by adjusting the preform feed and fiber take-up rates. A selection of the investigated fiber samples was annealed at $100^{\circ} \mathrm{C}$ for 20 hours and the effect of annealing on differential stress optic coefficient and Young's modulus was also studied in this work.

\section{MESUREMENT OF STRESS-OPTIC COEFFICIENT}

To determine $\Delta \mathrm{C}=\mathrm{C}_{1}-\mathrm{C}_{2}$, we measured the dependence of fiber birefringence upon additional axial stress applied to the fiber, $\sigma_{\mathrm{z}}^{\text {applied. }}$. The drawing stress and geometrical parameters of the examined fibers are gathered in Table 1. To measure the fiber birefringence in the direction perpendicular to the symmetry axis, we used a system employing a Senarmont compensation principle. The system was composed of microscope objective, CCD camera, polarizer, motorized analyzer,

Photonic Crystal Fibers IV, edited by Kyriacos Kalli, Waclaw Urbanczyk, Proc. of SPIE Vol. 7714,

$77140 \mathrm{G} \cdot$ ? 2010 SPIE · CCC code: 0277-786X/10/\$18 · doi: 10.1117/12.855089 
achromatic quarterwave plate, and a bright LED source with central wavelength at $526 \mathrm{~nm}$, as it is shown in Fig. 1 . The investigated fibers were immersed in index matching liquid to avoid light refraction at the edges. Intrinsic fiber birefringence arising during the drawing process or introduced by applied axial stress, causes retardation between wavefronts of orthogonal polarizations $\Sigma_{\mathrm{x}}(\mathrm{x})$ and $\Sigma_{\mathrm{y}}(\mathrm{x})$, when the fiber is illuminated perpendicularly to its symmetry axis. To determine the retardation distribution across the fiber $\mathrm{R}(\mathrm{x})=\Sigma_{\mathrm{x}}(\mathrm{x})-\Sigma_{\mathrm{y}}(\mathrm{x})$, a computer controlled CCD camera took a series of pictures for different positions of a rotatable analyzer. After appropriate processing of a series of collected photographs, the retardation maps were obtained for all investigated fibers.

Table 1. Parameters of the investigated fibers. All annealed fibers are indicated by an apostrophe. Annealing temperature was $100^{\circ} \mathrm{C}$ and duration $20 \mathrm{~h}$.

\begin{tabular}{|c|l|c|c|c|}
\hline $\begin{array}{l}\text { Fiber } \\
\text { sample }\end{array}$ & $\begin{array}{c}\text { Measured } \\
\text { diameter } \\
{[\mathrm{mm}]}\end{array}$ & $\begin{array}{c}\sigma_{\text {drawing }} \\
{[\mathrm{MPa}]}\end{array}$ & $\begin{array}{c}\text { Measured } \Delta \mathrm{C} \\
\mathrm{Pa}^{-1}\end{array}$ & $\begin{array}{c}\text { Measured initial } \\
\text { birefringence }\end{array}$ \\
\hline $1 \mathrm{a}^{\prime}$ & 0.605 & 2.0 & $-4.3 \times 10^{-12}$ & $-1.05 \times 10^{-4}$ \\
\hline $1 \mathrm{a}$ & 0.556 & 2.3 & $-4.37 \times 10^{-12}$ & $-1.44 \times 10^{-4}$ \\
\hline $1 \mathrm{~b}^{\prime}$ & 0.329 & 8.1 & $-3.45 \times 10^{-12}$ & $-3.71 \times 10^{-4}$ \\
\hline $1 \mathrm{~b}$ & 0.387 & 5.9 & $-3.62 \times 10^{-12}$ & $-3.23 \times 10^{-4}$ \\
\hline $1 \mathrm{c}^{\prime}$ & 0.126 & 19.0 & $-2.78 \times 10^{-12}$ & $-5.61 \times 10^{-4}$ \\
\hline $1 \mathrm{c}$ & 0.105 & 27.2 & $-1.54 \times 10^{-12}$ & $-7.91 \times 10^{-4}$ \\
\hline $1 \mathrm{~d}^{\prime}$ & 0.396 & 10.4 & $-3.20 \times 10^{-12}$ & $-4.19 \times 10^{-4}$ \\
\hline $1 \mathrm{~d}$ & 0.294 & 18.7 & $-1.79 \times 10^{-12}$ & $-7.91 \times 10^{-4}$ \\
\hline $1 \mathrm{e}^{\prime}$ & 0.291 & 18.8 & $-3.16 \times 10^{-12}$ & $-5.27 \times 10^{-4}$ \\
\hline $1 \mathrm{e}$ & 0.263 & 23.1 & $-1.78 \times 10^{-12}$ & $-8.18 \times 10^{-4}$ \\
\hline
\end{tabular}

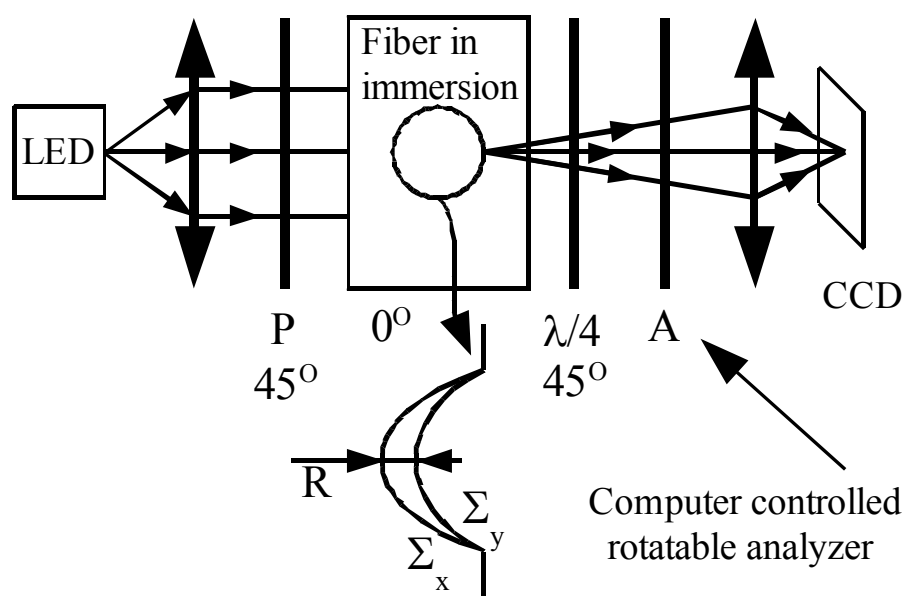

Fig.1. Experimental set-up for measuring retardation distribution in the PMMA fibers.

The measured retardation distribution $\mathrm{R}(\mathrm{x})$ is related to the axial stress distribution $\sigma_{\mathrm{z}}(\mathrm{r})$ in the fibers cross-section by the inverse Abel transformation [5]:

$$
\sigma_{z} \Delta C=\frac{-1}{\pi} \int_{r}^{r_{0}} \frac{d R(x) / d x}{\sqrt{x^{2}-r^{2}}} d x
$$


where $\mathrm{r}_{0}$ is the fiber radius, $\sigma_{z}(r)$ is the axial stress, and $\mathrm{dR} / \mathrm{dx}$ is a derivative of measured retardation. The differential stress optic coefficient $\Delta C$ can be easily retrieved by applying increasing axial load to the fiber and finally by plotting the dependence $\Delta C \sigma_{z}^{\text {calculated }}$ versus $\sigma_{z}^{\text {applied }}$ with $\Delta C$ being a proportionality coefficient.

An axial stress applied to the fiber induces optical anisotropy characterized by three principal indices $n_{z}, n_{r}, n_{\theta}$. As it is shown in earlier publication [5], when axial stress is applied to a fiber made of uniform material, other stress components in the fiber cross section are significantly lower than $\sigma_{z}\left(\sigma_{z}>>\sigma_{r} \quad \sigma_{z} \gg>\sigma_{\Theta}\right)$, which results in the following relation:

$$
\sigma_{z}(r) \Delta C=n_{z}(r)-n_{\Theta}(r) .
$$

As the investigated fibers are made of uniform PMMA, the inverse Abel transformation of the measured retardation distribution $\mathrm{R}(\mathrm{x})$ provides directly information about the birefringence induced by applied axial stress.

To test our measurement and processing method, we first measured $\Delta C$ in a reference silica fiber. Silica is a very well characterized material thus allowing us to compare the results obtained using our method with a reliable literature value [6], $\Delta C=(3.57 \pm 0.03) \times 10^{-12} \mathrm{~Pa}^{-1}$ at $526 \mathrm{~nm}$. In Fig.2 phase retardation maps are shown for unloaded and loaded fiber. In Fig. $3 \mathrm{~b}$ we show the birefringence distribution reconstructed from the retardation maps measured for different axial stress applied (Fig.3a).

(a)

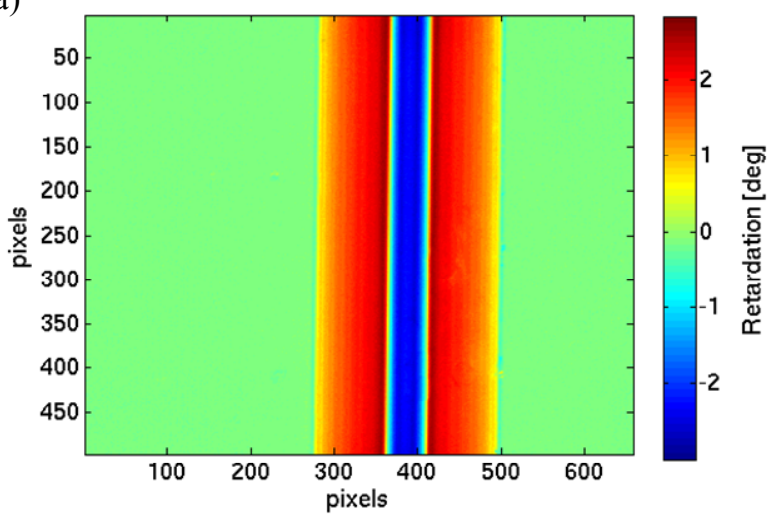

(b)

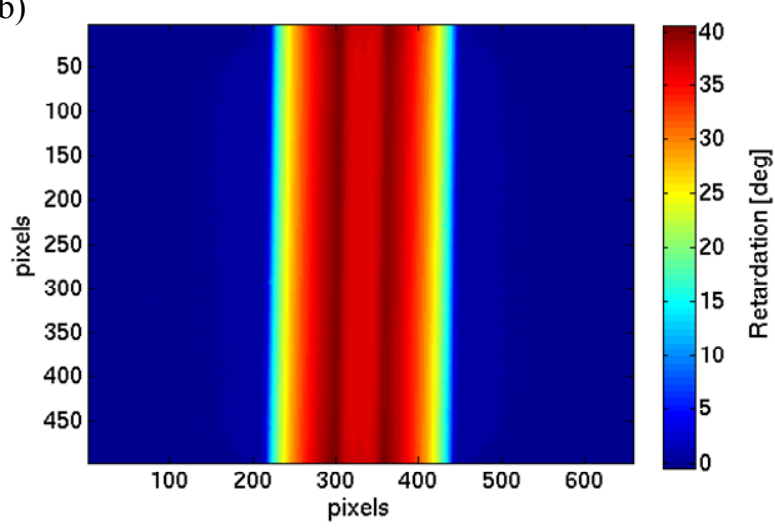

Fig.2. Phase retardation map measured in silica fiber in transverse direction: no stress applied (a), 133 MPa stress applied (b).

(a)

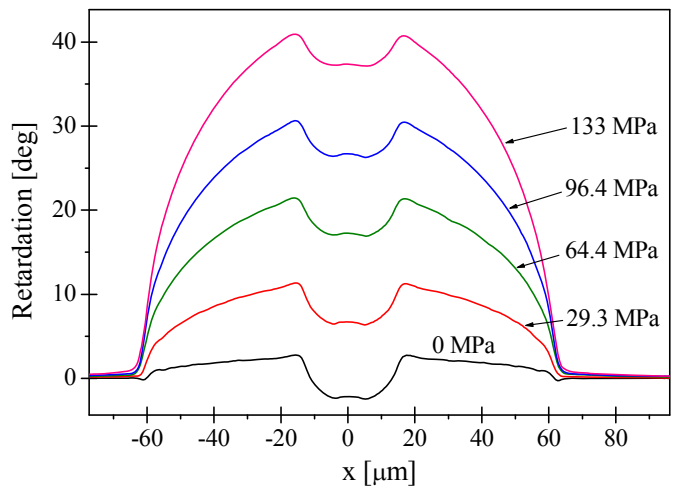

(b)

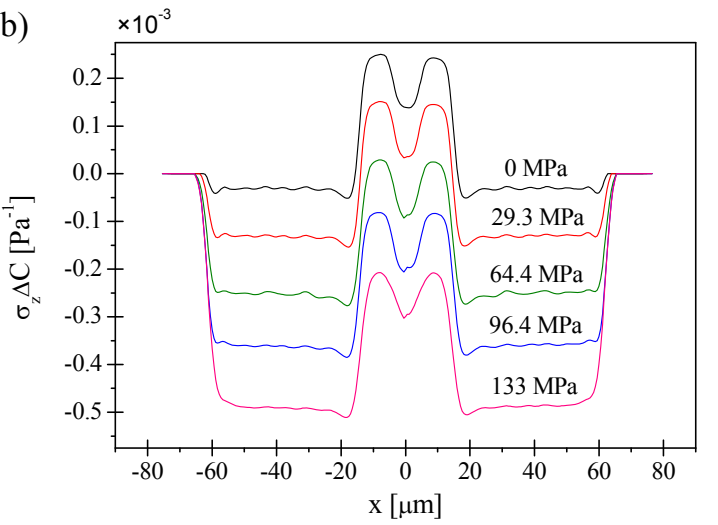

Fig.3. Phase retardation measured in silica fiber for different axial stress applied (a) and birefringence reconstructed using the inverse Abel transform (b).

By the linear fitting of reconstructed birefringence in pure silica cladding versus applied stress, we obtained a value for the stress-optic coefficient of $\Delta \mathrm{C}=(3.52 \pm 0.01) \times 10^{-12} \mathrm{~Pa}^{-1}$, see Fig.6a. The measured value is almost the same as the one reported in literature, which gives confidence in our measurement and processing methods.

Similar measurements were performed for the PMMA fibers drawn with different stress (Table 1). In Fig.5b we, 
show the birefringence distribution reconstructed from the retardation measured for different axial stress applied (Fig.5a), $\sigma_{z}^{\text {applied }}$ in the PMMA fiber indicated as $1 \mathrm{~b}$, which was drawn using a relatively small drawing stress of $5.85 \mathrm{MPa}$.

(a)

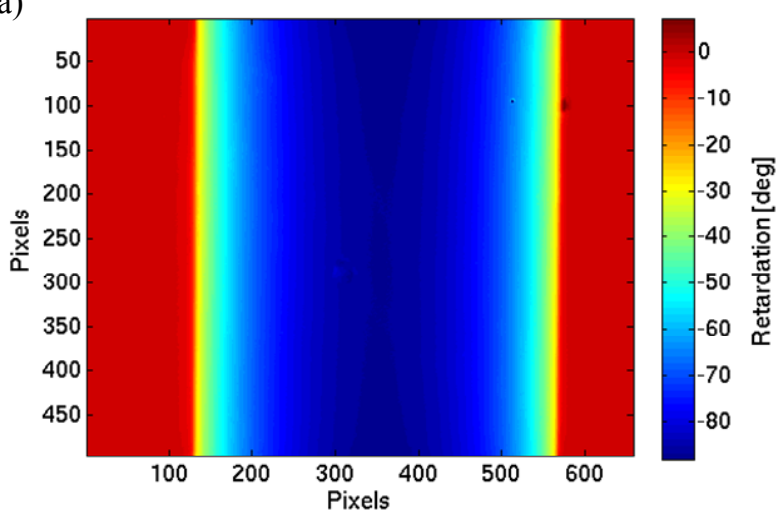

(b)

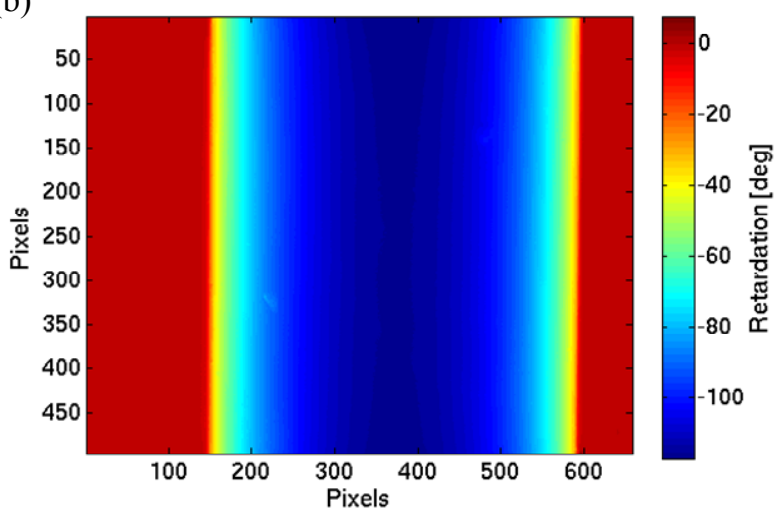

Fig.4. Phase retardation map measured in 1b PMMA fiber in transverse direction for applied axial stress of $2.62 \mathrm{MPa}$ (a) and $30.9 \mathrm{MPa}(\mathrm{b})$.

(a)

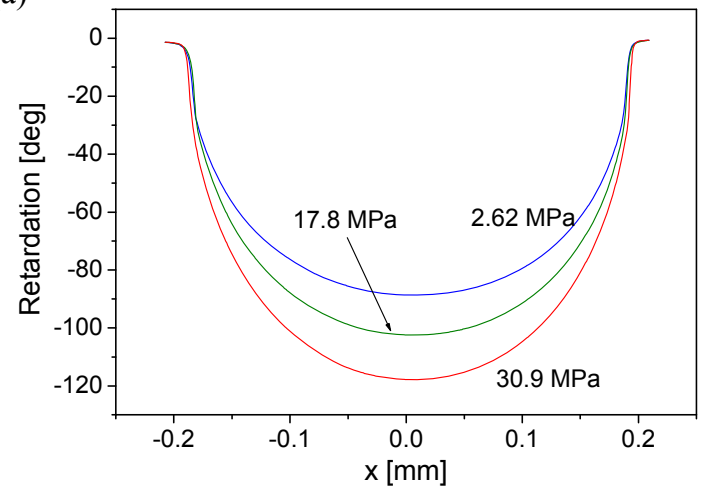

(b)

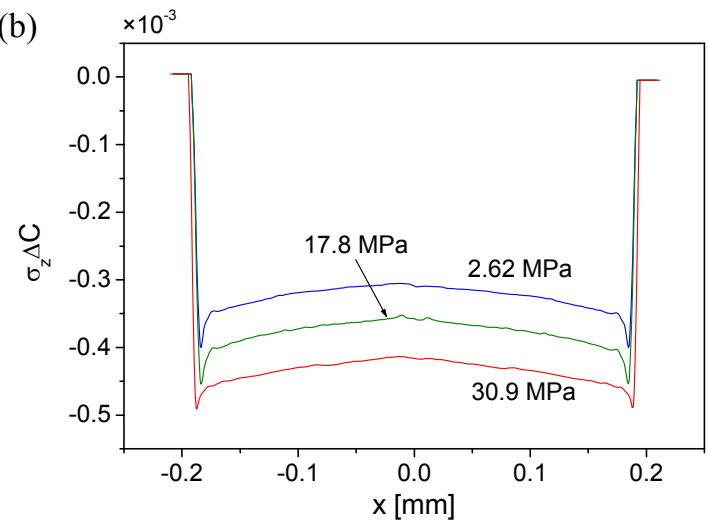

Fig.5. Phase retardation measured in $1 \mathrm{~b}$ PMMA fiber for different axial stress applied (a) and birefringence reconstructed using the inverse Abel transform (b).

For all investigated PMMA fibers, the applied axial stress was gradually increased until the fiber broke. As it can be clearly seen in Fig.5b, the reconstructed distribution of stress-induced birefringence $\Delta C \sigma_{z}$ is not perfectly uniform in the fibers' cross-sections. An increase of $\Delta C \sigma_{z}(\mathrm{r})$ near the fiber edges by about $10 \%$ is most probably caused by the higher cooling rate of the external layers of the fiber during the drawing process. To determine $\Delta C$ in the polymer fibers, we averaged the measured distribution of $\Delta C \sigma_{z}(\mathrm{r})(\mathrm{r})$ in the fiber cross-section and plotted the dependence of the averaged $\sigma_{z}(\mathrm{r}) \Delta C=\mathrm{n}_{\mathrm{z}}-\mathrm{n}_{\Theta}$ as a function of applied stress, see Fig.6a. In order not to obscure this figure, we show only the results obtained for a few fibers having significantly different $\Delta C$. 

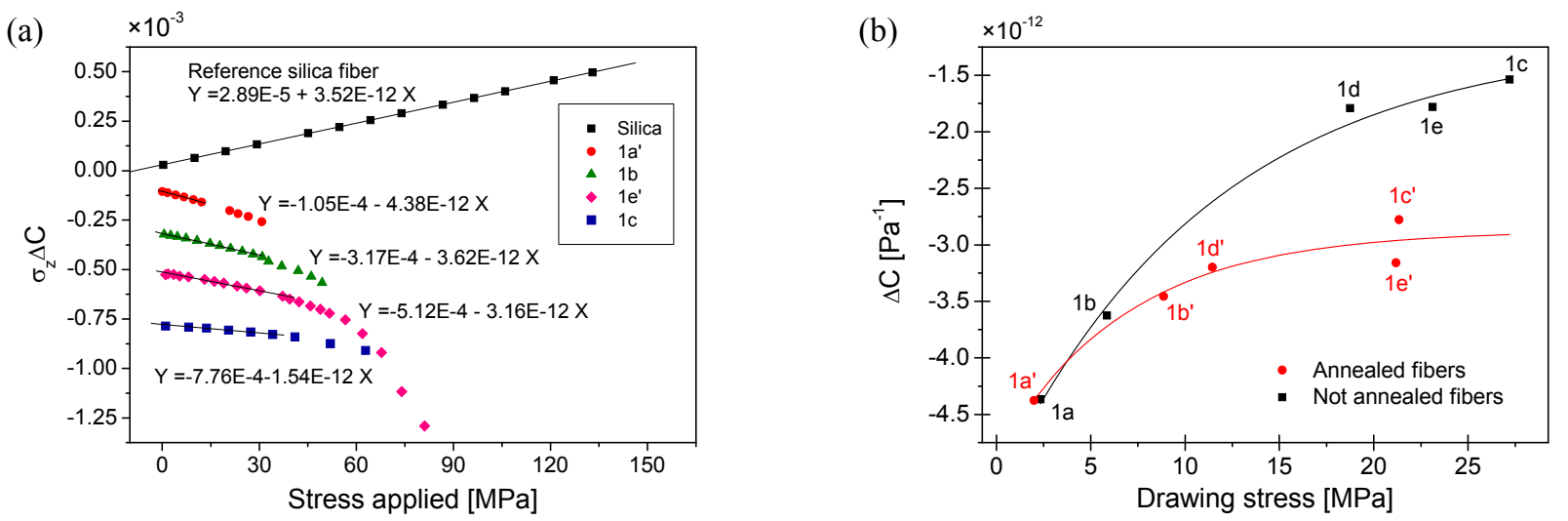

Fig.6. Birefringence versus applied stress for silica and PMMA fibers (a) and $\Delta C$ versus drawing stress for PMMA fibers (b).

The birefringence induced by applied axial stress increases linearly for small values of $\sigma_{z}$ applied. The stress optic coefficient $\Delta C$ was determined for each PMMA fiber by linear approximation of the dependence of $\Delta C \sigma_{z}^{\text {calculated }}$ (averaged over the fiber cross-section) versus $\sigma_{z}^{\text {applied }}$ for small values of $\sigma_{z}^{\text {applied }}$, with $\Delta C$ being a regression coefficient. Finally, the dependence of the stress-optic coefficient $\Delta C$ upon drawing stress is presented in Fig.6b. The value of $\Delta C$ increases against $\sigma_{\text {drawing }}$ from -4.5 to $-1.5 \times 10^{-12} \mathrm{~Pa}^{-1}$ in a nonlinear manner and appears to gradually saturate for high drawing stress. The same measurements were repeated for the fibers annealed at a temperature of $100^{\circ} \mathrm{C}$ for 20 hours. It is interesting that fiber annealing does not change the general trend in the dependence of $\Delta C$ versus $\sigma_{\text {drawing }}$, however the saturation effect for annealed fibers occurs at a greater absolute value of $\Delta C$. Next, we show in Fig.7 the dependence of $\Delta \mathrm{C}$ upon residual fiber birefringence, which is clearly linear with no significant impact of the annealing process.

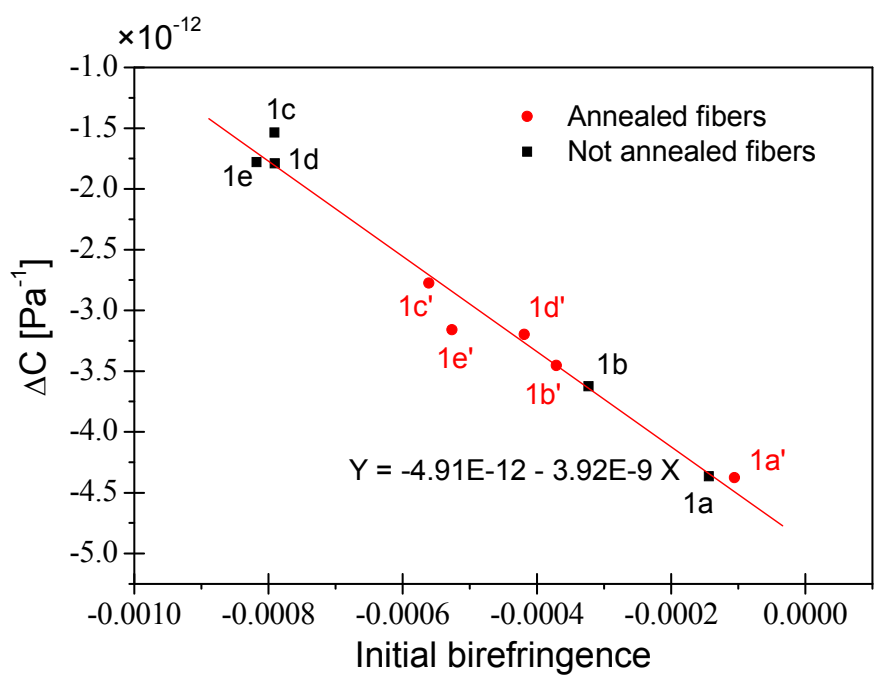

Fig.7. Dependence of the stress optic coefficient on initial fiber birefringence

This experimental result shows that the absolute value of the stress optic coefficient $\Delta \mathrm{C}$ decreases in the PMMA fibers having greater initial birefringence, which directly corresponds to a greater initial ordering of molecules. As the trend line shown in Fig. 9 takes a value of $-4.8 \times 10^{-12} \mathrm{~Pa}^{-1}$ at zero birefringence, we can estimate $\Delta \mathrm{C}$ for the bulk PMMA used to draw the fibers, $\Delta \mathrm{C}=-4.8 \times 10^{-12} \mathrm{~Pa}^{-1}$. 


\section{MESUREMENT OF YOUNG'S MODULUS}

The experimental arrangement shown in Fig. 8 was employed to measure Young modulus in the investigated PMMA fibers. This system is based on a digital image correlation (DIC) technique using one camera and allows measurement of the strain-stress curves. The magnified image of one sample of the POF obtained by the DIC camera using a microscope with a $3.5 x$ objective is shown in Fig.9a.

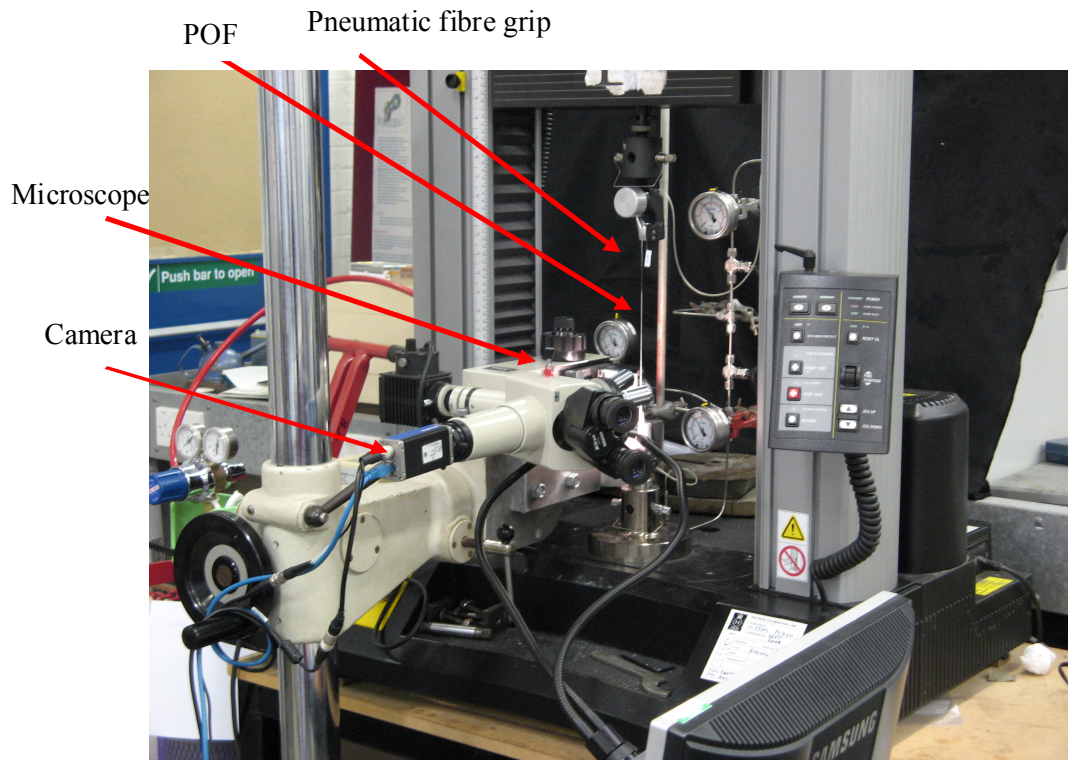

Fig.8. Experimental set-up for measuring Young's modulus in PMMA fibers.
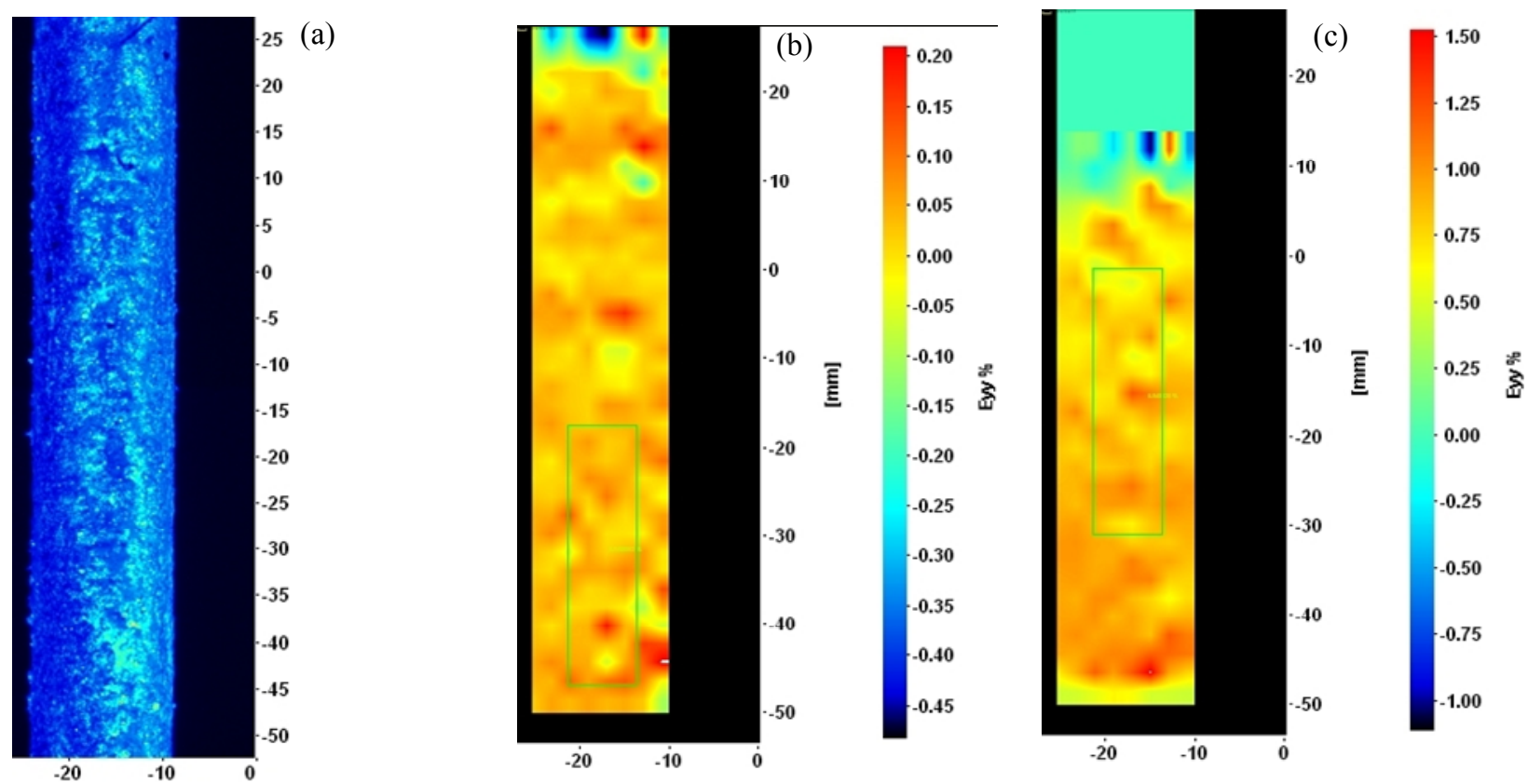

Fig.9. Image of POF sample indicated as 1a (a), strain map obtained for stress applied $\sigma_{z}^{\text {applied }}=3 \mathrm{MPa}$ (b), strain map obtained for $\sigma_{z}^{\text {applied }}=28 \mathrm{MPa}(\mathrm{c})$. 
The length of fiber within the image frame was approximately $3 \mathrm{~mm}$. Note that images shown in Fig.9b and c have different scales. To facilitate the DIC measurement, the POFs were covered with white spray paint and some black powder. Strain maps of the POF sample with different stresses, as obtained from DIC, are shown in Fig.9b-c. Representative stress-strain curves for samples 1e and 1e' are shown in Fig.10. The key influence on the accuracy of the calculated stress and Young's modulus is the uncertainty of the diameter of the POF. The diameters of the POFs were non-uniform along the sample length due to lack of diameter control in the drawing process. In addition, the cross sections of some fibres were not quite circular. A digital micrometer was used to measure the diameter of the fibres. The diameter used to calculate applied stress was averaged over the section of fibre where DIC images were taken. The typical error of diameter is estimated at $4 \mu \mathrm{m}$. Young's modulus values calculated from the stress-strain curves are plotted against the fibre drawing tension in Fig.11.

Linear regression applied to the data suggests that drawing stress may influence the Young's modulus, with greater stress leading to a higher modulus. It should be noted however that the gradient of the line of best fit is $0.017 \mathrm{GPa} / \mathrm{MPa}$ while the standard error in the gradient is almost half this value at $0.008 \mathrm{GPa} / \mathrm{MPa}$, so there is some uncertainty as to whether there really is a correlation, as reflected by the correlation coefficient of only 0.37 . Further research is needed to confirm if the two quantities are correlated, but even if they are, control over the elastic modulus is only likely to be over a range of perhaps $20 \%$ of its nominal value. The data in Fig. 11 also suggest that the annealing process does not have any significant impact on the Young's modulus.

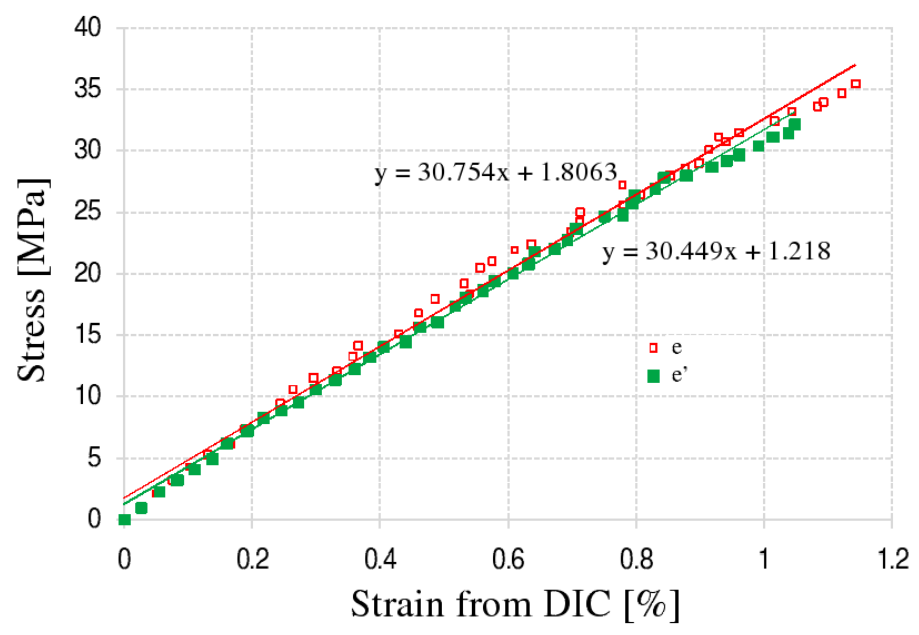

Fig.10. Stress-strain curves measured for POF indicated as 1e and 1e'.

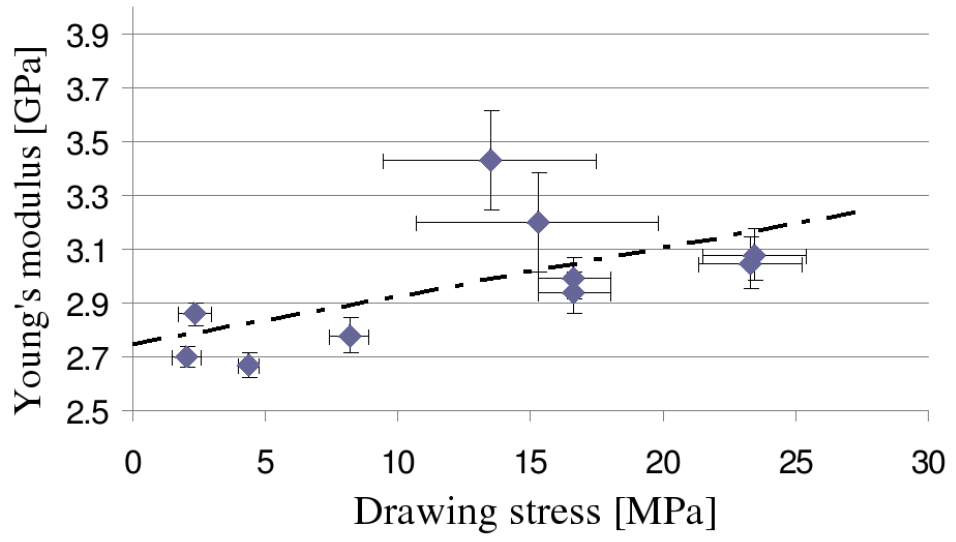

Fig.11. Dependence of Young's modulus upon drawing stress. 


\section{CONCLUSIONS}

From the presented series of experiments we conclude that the stress-optic coefficient $\Delta \mathrm{C}$ in PMMA fibers has negative sign and with our fibers ranges from -4.5 to $-1.5 \times 10^{-12} \mathrm{~Pa}^{-1}$ depending on the drawing stress. Dependence of $\Delta \mathrm{C}$ is

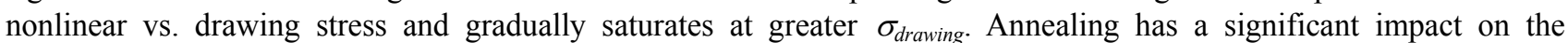
dependence of the differential stress-optic coefficient upon $\sigma_{\text {drawing. }}$. Moreover, our measurements demonstrate that $\Delta \mathrm{C}$ is linearly proportional to initial fiber birefringence with annealing having no impact on the slope of this dependence. From the DIC measurements, we conclude that Young's modulus may only slightly depend on POF drawing stress. More accurate measurements are necessary to confirm that result. Moreover, annealing doesn't have impact on Young's modulus value.

Acknowledgements: The work is partly supported by the EU FP7 project PHOSFOS and the Statutory Grant at Wroclaw University of Technology. M. Szczurowski, G. Satkiewicz-Barabach and W. Urbanczyk acknowledge support of the FNP Program "MISTRZ".

\section{REFERENCES}

[1] Ohkita, H., Ishibashi, K., Tsurumoto, D., Tagaya A., Koike, Y., "Compensation of the photoelastic birefringence of a polymer by doping with an anisotropic molecule", Applied Physics A: Materials Science and Processing 81, $617-620$ (2005).

[2] Koyama, T., Zhu, Y., Otsuka, T., Takada, T., Murooka, Y., “An automatic measurement system for 2-dimensional birefringence vector distribution”, Proc. IEEE, 557 - 560 (1998).

[3] Xu, W., Yao, X. F., Yeh, H. Y., Jin, G. C., "Fracture investigation of PMMA specimen using coherent gradient sensing (CGS) technology", Polymer Testing 24, 900 - 908 (2005).

[4] Waxler, R. M., Horowitz, D., Feldman, A., "Optical and physical parameters of Plexiglas 55 and Lexan", Applied Optics 18, 101 - 104 (1979).

[5] Urbańczyk, W., Pietraszkiewicz, K., "Measurements of stress anisotropy in fiber preform: modification of the dynamic spatial filtering technique", Applied Optics 27, 4117 - 4122 (1988).

[6] Primak, W., Post D., "Photoelastic constants of vitreous silica and its elastic coefficient of refractive index", J. of Appl. Physics 30, $779-788$ (1958). 